\title{
Крутов Р.А. \\ Преимущества и недостатки кредитного скоринга как метода оценки кредитоспособности потенциального заемщика
}

Финансовый университет при Правительстве РФ

(Россия, Москва)

doi: $10.18411 / 1 j-02-2021-85$

idsp: ljournal-02-2021-85

\section{Аннотация}

В процессе кредитования наиболее серьезным этапом является оценка кредитоспособности. Именно грамотное определение кредитоспособности своих клиентов позволяет банкам преждевременно регулировать и минимизировать кредитные риски. Банки с течением времени применяли в целях определения кредитоспособности физических лиц и субъектов малого и среднего предпринимательства различные методики, все они обладают своими преимуществами и недостатками, но на сегодняшний день с развитием информационных и компьютерных технологий наиболее популярной и эффективной стала скоринговая методика.

Ключевые слова: кредитный скоринг, кредитоспособность, оценка кредитоспособности, скоринговые методики, преимущества и недостатки скоринга.

\section{Abstract}

The most important stage in the lending process is the assessment of creditworthiness. It allows banks to prematurely regulate and minimize credit risks. Over time, banks have used various methods to determine the creditworthiness of individuals and small and medium-sized businesses, all of them have their own advantages and disadvantages, but today, with the development of information and computer technologies, the scoring method has become the most popular and effective.

Keywords: credit scoring, creditworthiness, credit rating, scoring techniques, advantages and disadvantages of credit scoring.

Кредитная деятельность для любого коммерческого банка является основополагающей и, с одной стороны, приносит значительную часть доходов, а с другой - может привести при неблагоприятных условиях к банкротству кредитного учреждения в связи с невозвратом высокой доли кредитов, особенно крупных. Проблема существования кредитного риска приводит к тому, что коммерческие банки должны искать различные способы и методы снижения воздействия этого риска во избежание потери прибыли и понесения чрезмерно больших убытков вследствие превращения кредитного риска в реальные невозвращенные кредиты. Здесь большое значение начинает иметь понятие кредитоспособности заемщика и необходимость ее определения коммерческими банками.

Оценка кредитоспособности представляет собой один из этапов процесса управления кредитным риском, являясь качественной оценкой способности заемщика рассчитываться по своим обязательствам с банком. Кредитные учреждения при проведении оценки кредитоспособности потенциальных заемщиков своей конечной целью ставят определение возможности и готовности кредитополучателя погашать все свои обязательства перед кредитной организацией в соответствии с условиями кредитного договора. Но помимо достижения этой конечной цели, кредитные учреждения путем проведения оценки кредитоспособности стремятся также решить следующие задачи: 
- Изучение финансового состояния заемщика и определение факторов, способных впоследствии каким-либо образом повлиять на качество финансового состояния в лучшую, или, что важнее, в худшую сторону;

- Предупреждение и минимизация потерь денежных средств, выданных в кредит, в результате нежелания и/или неспособности заемщика выплатить сумму основного долга и начисленных процентов;

- Определение конкретного результативного показателя кредитоспособности заемщика, позволяющего сравнивать заемщиков друг с другом, анализировать принятые на себя кредитные риски и корректировать кредитную политику.

Существует достаточно большое количество различных методик, позволяющих оценить кредитоспособность заемщика (см. Таблицу 1). Здесь стоит отметить, что Центральный банк Российской Федерации на сегодняшний день никакими нормативными документами не утверждает в обязательном порядке применение конкретной методики определения кредитоспособности, поэтому российские банки занимаются созданием собственных методик, опираясь на опыт зарубежных банков и адаптируя их под российскую реальность.

\begin{tabular}{|c|c|}
\hline Группа методик & Примеры методик \\
\hline \multirow{2}{*}{ Рейтинговые методы } & Скоринговые (Д. Дюрана) \\
\hline & Матричные (Модель Г.В. Савицкой) \\
\hline $\begin{array}{l}\text { Методы прогнозирования } \\
\text { банкротств }\end{array}$ & $\begin{array}{l}\text { Множественный дискриминантный анализ (Z-модели Альтмана, Лиса, } \\
\text { Тафлера, Бивера, Фульмера, М.А. Федоровой, Г. Спрингейта, Р.С. } \\
\text { Сайфулина и Г.Г. Кадыкова, О.П. Зайцевой, Л.В. Донцовой и Н.А. } \\
\text { Никифировой, Модель R; PAS - коэффициенты); Логит-модели } \\
\text { (модели Чессера, Г.В. Савицкой) }\end{array}$ \\
\hline \multirow{2}{*}{ Регрессионные методы } & Метод Аргенти (А-счет) \\
\hline & Регрессионные деревья (CART) \\
\hline $\begin{array}{l}\text { Методики качественного } \\
\text { анализа }\end{array}$ & $\begin{array}{l}\text { CAMPARI, PARTS, правила «пяти Си» и «шести Си», PARSER, } \\
\text { CAMEL, COPF, методика АРБ. }\end{array}$ \\
\hline
\end{tabular}

Таким образом, становится возможным сделать следующий вывод: оценка кредитоспособности заемщика необходима для коммерческих банков, поскольку позволяет говорить об уровне финансовой стабильности и устойчивости кредитополучателя, а значит, сделать вывод о том, сможет ли он погасить все свои обязательства в срок, и не возникнет ли в дальнейшем такой ситуации, когда банку придется окончательно смириться с фактом потери выданных в кредит денежных средств; кроме того, грамотная оценка финансового состояния и кредитоспособности заемщика позволяет правильно определить категорию качества выданной заемщику ссуды и создать необходимый резерв на возможные потери по этой ссуде. Оценка кредитоспособности - это важнейший этап системы управления кредитным риском, позволяющий впоследствии принять верные решения в целях минимизации этого риска, а поскольку кредитный риск является наиболее значимым в деятельности любого банка, то пренебрежение качеством и эффективностью его управления на отдельных этапах может привести к последствиям различного масштаба.

В конечном счете, несмотря на то, какую конкретно методику оценки кредитоспособности заемщика применяет коммерческий банк, в результате оценки кредитное учреждение должно вынести правильное заключение о надежности потенциального кредитополучателя, не упуская в процессе принятия решения влияние различных деталей и множества факторов, способных в той или иной степени изменить финансовое состояние заемщика и привести к образованию проблемных кредитов.

При определении кредитоспособности потенциального заемщика необходимо учитывать все аспекты, которые исходят из принципов кредитования - платности, 
срочности и возвратности. Таким образом, необходимо обращать внимание не только на то, что заемные средства должны быть возвращены заемщиком полностью и в установленный кредитным договором срок, но и на то, что помимо суммы основного долга необходимо выплатить и проценты по кредиту. Далее необходимо учесть все детали, характеризующие способность заемщика покрыть все свои обязательства по кредиту.

При этом, если мы говорим о традиционных экспертных подходах к оценке кредитоспособности, они несмотря на свою относительно достаточную работоспособность и эффективность, одновременно с этим, имеют ряд проблем:

- Мнение специалистов, проводящих оценку, субъективно. Таким образом, точность оценки и её качество сильно зависит от знаний, опыта и профессионализма кредитных специалистов.

- Сотрудники физически неспособны обрабатывать огромные объемы информации, а значит - это приводит к проблеме ограниченности числа рассматриваемых банком кредитных заявок.

- Квалификация сотрудников, проводящих анализ кредитоспособности потенциального заемщика, должна быть достаточно высока; такие навыки должны быть высоко оценены в вопросе заработной платы, что приводит к значительным расходам банка.

В связи с обозначенными выше проблемами, становится понятной и очевидной позиция коммерческих банков, заинтересованных в создании таких систем классификации кредитов и анализа кредитоспособности заемщиков, которые могли бы позволить свести к минимуму участие экспертов в принятии решений в области кредитного анализа, и таким образом, уменьшить влияние человеческого фактора.

В такой ситуации на помощь коммерческим банкам приходит скоринговая методика оценки кредитоспособности, позволяющая оценить не только реальное финансовое состояние заемщика, но и его кредитный потенциал. Важное отличие кредитного скоринга от экспертной оценки заключается в том, что работа скоринговой системы основана на математическом и статистическом анализе кредитной истории «прошлых» заемщиков банка, при этом лишена субъективности, присущей человеку.

Упрощенно, скоринг - это начисление очков или баллов за конкретный критерий и выведение общего кредитного рейтинга на основе суммы всех начисленных баллов, позволяющего сделать вывод о возможности выдачи или невыдачи кредита. Любая скоринговая система подразумевает в своей работе три основных этапа: сбор информации, её анализ и выдача результата по итогам анализа. Сбор информации - это введение всех необходимых данных, критериев и параметров, присущих заемщику на основе предоставленных им документов, заполненной анкеты и информации из бюро кредитных историй и некоторых государственных органов. Анализ подразумевает начисление баллов за каждый критерий с учетом веса, то есть с учётом важности конкретного критерия для коммерческого банка. И наконец, на последнем этапе все баллы суммируются и выводится кредитный рейтинг, характеризующий кредитоспособность заемщика и дающий возможность принять решение о выдаче кредита клиенту. В модели существуют числовые границы, в рамках которых вычисляется кредитный рейтинг, а также присутствует так называемая линия отсечения. Для того, чтобы получить кредит в банке, необходимо набрать баллы выше линии отсечения, таким образом банки и отсекают ненадежных, по их мнению, заемщиков.

Таким образом, коммерческие банки, применяя скоринговую систему оценки кредитоспособности заемщиков, решают следующие проблемы, которые возникают при использовании традиционных подходов: 
- субъективизм;

— негибкость и нестабильность;

- отсутствие системы обучения и накопления знаний;

- ограничение числа рассматриваемых заявок, которое обусловлено объективно ограниченными физическими ресурсами человека.

Помимо существующих преимуществ кредитного скоринга, у него имеются и свои недостатки. Одна из проблем связана с тем, что для построения качественной скоринговой модели необходимо обладать относительно большим количеством данных о поведении клиентов. При этом использовать уже имеющиеся данные, но принадлежащие другим банкам, или только данные, полученные из свободных источников, не совсем правильно, так как в этом случае возникает проблема сопоставимости и качества исходных данных, поскольку поведение заемщиков в разных банках может сильно отличаться, тем более в тех случаях, когда речь идет о банках разной клиентоориентированности в разных городах и регионах. Таким образом, модель, составленная на основе данных о работе с клиентами в одном банке, вероятно, не будет также эффективно работать в другом банке. Соответственно, банки, не обладающие собственной большой клиентской базой, не смогут с высокой долей вероятности разработать и внедрить собственную качественную и работоспособную скоринговую систему, не прибегая к помощи сторонних фирм, работающих в области кредитного скоринга.

Вторая серьезная проблема применения кредитного скоринга заключается в том, что все знания, представленные в виде скоринговых моделей, не имеют чёткой логики, поскольку само их построение основано лишь на поиске статистических закономерностей. Модель, основанная на таких технологиях, будет находить и отражать все найденные статистические закономерности, однако при попытке рационально объяснить корреляцию между признаками перед программистом или кредитным специалистом в лучшем случае возникнут существенные трудности, а в худшем - задача окажется нерешаемой. На сегодняшний день технологии машинного обучения усугубляют эту ситуацию, и ни одна модель, основанная на технологиях с применением искусственного интеллекта и машинного обучения, никогда не сможет объяснить почему она конкретному заемщику присвоила один класс кредитоспособности, а второму - другой.

Тем не менее несмотря на имеющиеся недостатки и на то, какие технологии применяются непосредственно в самой модели кредитного скоринга, любой коммерческий банк, внедряя скоринговые методики, рассчитывает на получение нескольких преимуществ по сравнению с прочими метода оценки кредитоспособности:

- возможность минимизировать затраты и снизить операционные риски за счёт автоматизированного процесса оценки кредитоспособности и принятия решения о кредитовании;

- сокращение временных затрат при обработке кредитных заявок;

- увеличение величины обрабатываемых заявок;

- отсутствие влияния субъективных факторов при принятии решения.

Таким образом, скоринговые методики являются на сегодняшний день наиболее эффективным решением в области определения кредитоспособности как физических лиц, так и субъектов малого и среднего предпринимательства. Грамотное использование банками кредитного скоринга выводит их на более высокий конкурентоспособный уровень на российской и международной арене, позволяет минимизировать отток «хороших» клиентов и наиболее быстрым способом избавляться 
от «плохих», а также это открывает новые возможности в будущем в рамках внедрения ЕСИА и ЕБС и развития полноценного дистанционного банковского обслуживания.

$$
* * *
$$

1. Положение Банка России от 28 июня 2017 г. № 590-П «Положение о порядке формирования кредитными организациями резервов на возможные потери по ссудам, ссудной и приравненной к ней задолженности» (с послед. изм.) // СПС КонсультантПлюс.

2. Ачкасов А.И. Активные операции коммерческих банков / А.И. Ачкасов. - М.: Консалт-Банкир, 2009. - с. 35

3. Кирисюк Г.М. Оценка банком кредитоспособности заемщика// Деньги и кредит. - 2010. - № 4. c.32;

4. Лаврушин, О.И. Банковское дело: Учебник. - 11-е изд., перераб. и доп. / Под ред. О.И.Лаврушина. - М.: Финансы и статистика, 2014. - 672c;

5. Сахарова М.О. К вопросу о кредитоспособности предприятия // Деньги и кредит. - 2009. - №3. - с. 20;

6. Севрук В.Т. Анализ кредитоспособности СП // Деньги и кредит, - 2009. - №3. - с. 43.

Лосева А.С., Гриднева Ю.С., Дёмина В.О. Организация системы внутреннего контроля на предприятии

ФГБОУ ВО Мичуринский государственный аграрный университет

doi: $10.18411 / \mathrm{lj}-02-2021-86$

(Россия, Мичуринск)

idsp: ljournal-02-2021-86

\section{Аннотация}

В статье рассматриваются особенности организации и функционирования системы внутреннего контроля на предприятиях с учетом влияния конкурентной среды. Показаны компоненты внутреннего контроля, обоснована внутренняя организационная структура центра внутреннего контроля на предприятиях. Определены основные направления действия внутреннего контроля на предприятии, проявляющиеся по его ключевым объектам, планируемым мероприятиям и контрольным процедурам.

Ключевые слова: контроль, система внутреннего контроля, внешняя среда, внутренняя среда, контрольные процедуры

\section{Abstract}

The article examines the features of the organization and functioning of the internal control system at enterprises, taking into account the influence of the competitive environment. The components of internal control are shown, the internal organizational structure of the internal control center at enterprises is substantiated. The main directions of the internal control at the enterprise, which are manifested in its key objects, planned activities and control procedures, have been determined.

Key words: control, internal control system, external environment, internal environment, control procedures

В современных условиях хозяйствование любое предприятие независимо от своей организационно-правовой формы представляет собой открытую систему, имеющую безусловную хозяйственную независимость и функционирующую в условиях жесткой конкурентной среды.

Внутренним контролем называют особую организационную структуру, содержащую совокупность методов, приемов, способов, установленных руководством с целью устойчивого и динамичного развития хозяйствующего субъекта с условием соблюдения требований всех законодательных и нормативных актов [2].

По своей сути, контрольная среда представляет собой осознание руководством организации ценности важности системы внутреннего контроля, а также воздействия руководства организации по укреплению и поддержанию этой системы на конкретной предприятии [1]. 